\title{
Incorporating Acute HIV Screening into Routine HIV Testing at Sexually Transmitted Infection Clinics, and HIV Testing and Counseling Centers in Lilongwe, Malawi
}

\author{
Sarah E. Rutstein, PhD, *† Audrey E. Pettifor, PhD, MPH, $\neq$ Sam Phiri, PhD, MSc, $\S$ Gift Kamanga, PhD,\| \\ Irving F. Hoffman, PA, MPH, † Mina C. Hosseinipour, MD, MPH, † N Nora E. Rosenberg, PhD, MSPH, \\ Dominic Nsona, BA, § Dana Pasquale, MPH, $\neq$ Gerald Tegha, MTech,\| Kimberly A. Powers, MSPH, PhD, $\neq$ \\ Mcleod Phiri, BS, § Bisweck Tembo, $\S$ Wairimu Chege, MD, MPH, $q$ and William C. Miller, MD, PhD, MPH†f
}

\begin{abstract}
Background and Objectives: Integrating acute HIV-infection (AHI) testing into clinical settings is critical to prevent transmission, and realize potential treatment-as-prevention benefits. We evaluated acceptability of AHI testing and compared AHI prevalence at sexually transmitted infection (STI) clinics and HIV testing and counseling (HTC) clinics in Lilongwe, Malawi.
\end{abstract}

Methods: We conducted HIV RNA testing for HIV-seronegative patients visiting STI and HTC clinics. AHI was defined as positive RNA and negative/discordant rapid antibody tests. We evaluated demographic, behavioral, and transmission-risk differences between STI and HTC patients and assessed performance of a risk-score for targeted screening.

Results: Nearly two-thirds $(62.8 \%, 9280 / 14,755)$ of eligible patients consented to AHI testing. We identified 59 persons with AHI (prevalence $=0.64 \%$ )-a $0.9 \%$ case-identification increase. Prevalence was higher at STI $[1.03 \%(44 / 4255)]$ than at HTC clinics $[0.3 \%(15 / 5025), P<0.01]$, accounting for $2.3 \%$ of new diagnoses vs $0.3 \%$ at HTC clinic. Median viral load (VL) was 758,050 copies per milliliter; $25 \%(15 / 59)$ had VL $\geq 10,000,000$ copies per milliliter. Median VL was higher at STI $(1,000,000$ copies $/ \mathrm{mL})$ compared with HTC $(153,125$ copies $/ \mathrm{mL}, P=0.2)$. Among persons with AHI, those tested at STI clinics were more likely to report genital sores compared with those tested at HTC clinics $(54.6 \%$ vs $6.7 \%, P<0.01)$. The risk score algorithm performed well in identifying persons with AHI at HTC clinics (sensitivity $=73 \%$, specificity $=89 \%$ ).

Received for publication May 8, 2015; accepted September 4, 2015.

From the *Department of Health Policy and Management, University of North Carolina at Chapel Hill, Chapel Hill, NC; $\uparrow$ Division of Infectious Diseases, University of North Carolina at Chapel Hill, Chapel Hill, NC; $\$$ Department of Epidemiology, University of North Carolina at Chapel Hill, Chapel Hill, NC; §Lighthouse Trust, Lilongwe, Malawi; \|UNC Project, Lilongwe, Malawi; and IDivision of AIDS, National Institute of Allergy and Infectious Diseases, National Institutes of Health, Bethesda, MD.

Supported by NIH/NIAID R01 AI083059. S.E.R. was supported by NIH/ NIMH (F30 MH098731) and NIH/NIGMS (T32-GM008719).

Presented in part at AIDS 2014, July 21, 2014, Melbourne, Australia.

The authors have no conflicts of interest to disclose.

Correspondence to: Sarah E. Rutstein, PhD, Department of Health Policy and Management, University of North Carolina Chapel Hill, CB \#7411, Chapel Hill, NC 27599-7411 (e-mail: sarah_rutstein@med.unc.edu).

Copyright $(2015$ Wolters Kluwer Health, Inc. All rights reserved.
Conclusions: The majority of patients consented to AHI testing. AHI prevalence was substantially higher in STI clinics than HTC clinics. Remarkably high VLs and concomitant genital scores demonstrate the potential for transmission. Universal AHI screening at STI clinics, and targeted screening at HTC centers, should be considered.

Key Words: acute HIV infection, AHI screening, Malawi, HIV testing and counseling

(J Acquir Immune Defic Syndr 2016;71:272-280)

\section{INTRODUCTION}

Acute HIV-infection (AHI) is a 10-12 week period immediately after HIV acquisition, when HIV RNA is detectable but anti-HIV antibodies are not. Characterized by unrestricted replication of HIV and extremely high viral loads, persons with AHI are highly infectious ${ }^{1-5}$ and may be responsible for a substantial proportion of sexual and motherto-child HIV transmission. ${ }^{6-9}$ Failure to identify persons with AHI may compromise HIV prevention efforts, dampening the potential population-wide benefits of treatment-as-preventionthe use of early and universal antiretroviral therapy. ${ }^{10}$ This is especially important in Malawi which embraced a treatmentas-prevention approach in its prevention of mother-to-child transmission program in 2011 and is advancing this strategy through its 90-90-90 targets in its 2015-2020 National Strategic Plan.

Integrating feasible and acceptable AHI testing strategies into clinical settings in sub-Saharan Africa may be critical to prevent onward transmission and realize the potential patient and population benefits of treatment-as-prevention. In subSaharan Africa, nearly $40 \%$ of ongoing HIV transmission may be attributed to persons in the first 5 months of infection-many of whom would be missed using traditional antibody testing approaches. ${ }^{11}$ Without AHI testing, persons with AHI are told they are HIV-negative. Believing they are HIV-uninfected, they are more likely to continue engaging in high-risk sexual behaviors during the highly-infectious acute phase of infection, and may remain undiagnosed for extended periods. ${ }^{12-14}$

Despite the brevity of the period, the proportion of persons with AHI at the time of HIV testing is nontrivial, and screening for acute infection may be cost-effective. ${ }^{15-18} \mathrm{In}$ 
sub-Saharan Africa, 1\%-3\% of patients in outpatient clinics who met setting-specific clinical criteria had AHI. ${ }^{19-22}$ This prevalence varies with clinic setting. For example, among men visiting a dermatology clinic in Lilongwe, Malawi, $0.2 \%$ of HIV-seronegative patients were acutely infected, ${ }^{23}$ compared with $2 \%-5 \%$ of HIV-seronegative patients at a nearby sexually transmitted infection (STI) clinic. $^{23,24}$ The risk profiles of persons in these clinical settings determine AHI rates. Some patients seeking testing at HIV testing and counseling (HTC) clinics are at high risk for HIV infection, but may have different risk behaviors and biologic cofactors than their STI clinic counterparts, and the prevalence of AHI in this population is unknown. AHI prevalence and risk factors have never been compared in these 2 settings, to our knowledge.

In this article, we describe the AHI testing component of a pilot randomized controlled trial assessing behavioral and antiretroviral interventions during acute HIV infection. ${ }^{25}$ In this analysis, our objective was to evaluate patient acceptability of testing for AHI, and examine AHI prevalence, population characteristics, and risk profiles among patients seeking HIV testing at STI and HTC clinics in Lilongwe, Malawi. We also assessed performance of an existing risk score in predicting AHI among screened populations at each clinic. $^{26}$ Outcomes are designed to inform AHI screening strategies and priorities in sub-Saharan Africa.

\section{METHODS}

\section{Study Design and Setting}

We conducted a cross-sectional analysis to describe testing for AHI among HIV-seronegative patients in the context of a larger pilot study (ClinicalTrials.gov \#NCT01450189). We conducted AHI testing at 2 HTC and 2 STI clinics in Lilongwe, Malawi. Lilongwe, the capital city of Malawi, has a population of approximately 1 million persons and an estimated adult HIV prevalence of $17 \%{ }^{27}$ The 4 selected sites (Lighthouse HTC, Martin Preuss Centre HTC, Bwaila District Hospital STI, and Kamuzu Central Hospital STI clinics) are among the primary access points for HIV testing in Lilongwe. In total, the 2 HTC sites historically have served approximately 14,000 patients per year; the 2 STI clinics serve nearly 10,000 patients per year. Differences between these 2 clinic types include provision of syndromic management of STI symptoms (available at STI, not at HTC clinics) and colocation of ART services (available at HTC, not at STI clinics). Additionally, persons testing at HTC clinics are more likely to have been referred specifically for HIV testing from either inpatient units or a sexual partner, whereas this is less often the case at STI clinics where patients may be referred specifically for STI testing, but not necessarily HTC. Although not universally true, the HIV prevalence at HTC clinics in this evaluation are historically higher than prevalence rates observed at STI clinics in this study.

\section{Study Population and Eligibility}

As per Malawi national guidelines, opt-out HIV testing and counseling is conducted using a serial testing algorithm with Alere Determine HIV-1/2 (Alere, Inc, Waltham, MA) and Uni-Gold Recombigen HIV-1/2 (Trinity Biotech, Bray, Ireland) rapid antibody tests. A tie-breaker is used in the event of discordant results.

Patients were eligible for AHI testing if they met the all the following criteria: (1) had negative or discordant rapid antibody test results, (2) were $\geq 18$ year old, (3) lived within the Lilongwe City catchment area, (4) were willing to provide locator information for follow-up tracing, and (5) were able to provide informed consent. Patients who had serious illness requiring inpatient management or were incarcerated were not eligible. Patient eligibility and consent were collected by HTC counselors after completion of routine HIV-antibody testing procedures and counseling. HIV prevalence was obtained from routine log data.

Acceptability of screening was assessed by the proportion of persons agreeing to AHI testing among those offered.

\section{Study Procedures}

We collected basic demographics, locater information, and AHI- and STI-symptom history (including presence of fever, diarrhea, or genital ulcers/sores) from all enrolled participants. Participants had $\sim 500 \mu \mathrm{L}$ of blood collected in microtubes by fingerstick. Specimens were stored in ice and transported daily to a central laboratory. Specimens were tested within 21 days of initial antibody testing; 98.8\% were tested within 7 days. All specimen and data collection was conducted by trained HTC counselors employed at the HTC and STI clinics.

\section{Laboratory Testing}

Specimens underwent HIV RNA polymerase chain reaction (PCR) testing using the COBAS AMPLICOR HIV-1 MONITOR Test (Roche, Pleasanton, CA) (reportable range of 50-750,000 copies/mL) or Abbott RealTime HIV-1 $m 2000$ Assay (Abbott Laboratories, Chicago, IL) (reportable range of 40-10,000,000 copies/mL). Microtubes containing whole blood were processed and plasma specimens were pooled in a 9:1 pooling algorithm. Positive pools were deconstructed and individual specimens tested to identify which contained detectable HIV RNA. ${ }^{28-31}$

\section{Definition of Acute HIV Infection}

Several definitions of AHI have been described; we selected that which was easiest to implement in a screening setting, focusing on markers of early infection that did not require extensive laboratory investigations. ${ }^{7}$ Specifically, AHI was defined as a positive HIV RNA test and negative or discordant ( 2 negative, 1 positive) rapid HIV antibody tests. When sufficient specimen was available, repeat antibody tests were conducted on samples with detectable HIV RNA to rule out initially false negative antibody results. Persons with positive HIV RNA were recontacted based on locator information provided at the time of testing, and counseled on their result. 


\section{Statistical Analyses}

All analyses were performed using Stata (Version 13.0; StataCorp, College Station, TX). We evaluated differences between HTC and STI patients by AHI status and testing site using $t$ tests, Pearson $\chi^{2}$ tests, and Fisher exact test with $\alpha=$ 0.05 . We used nonparametric Wilcoxon rank-sum tests to compare median viral loads (VLs).

\section{Evaluation of Existing Risk Score}

We also evaluated performance of an existing risk score algorithm developed to target AHI testing toward persons at highest risk. ${ }^{26}$ With this algorithm, each patient is assigned points for each of the following predictors: fever (1 point), body ache (1 point), having more than 1 sexual partner in the previous 2 months ( 1 point), diarrhea ( 2 points), genital ulcer disease ( 2 points), and discordant HIV rapid tests (4 points). Points are summed across predictors to calculate a risk score for each patient. Previous evaluations targeting AHI screening to those with a risk score $\geq 2$ suggest that $40.9 \%$ of all STI patients would meet this AHI testing threshold, and that 95.2\% of persons with AHI would be detected. We applied this score threshold to the entire tested study population, and evaluated the algorithm performance by clinic type.

\section{Ethical Approval}

The National Health Sciences Research Committee of Malawi, the Biomedical Institutional Review Board at University of North Carolina, Chapel Hill, and the National Institute of Allergy and Infectious Diseases Prevention Science Review Committee approved this study.

\section{RESULTS}

\section{Study Population and Acceptability}

Between June, 2012 and January, 2014, 35,913 patients received HTC at enrolling clinics (STI, $\mathrm{N}=13,379$; HTC, $\mathrm{N}=$ 22,525) (Fig. 1). HIV prevalence based on rapid antibody testing at enrolling clinics was 18.5\% (6647/35,913) (STI $14.0 \%$, HTC 21.2\%). Among the 29,257 HIV-antibodynegative or discordant patients, approximately half $(53.5 \%$, $15,655)$ were screened for study eligibility. The remaining $46.5 \%$ were not screened for eligibility and no additional demographic information is available. Overall $6 \%(900 / 15,655)$ were ineligible, due to age $<18(1.5 \%, 235 / 15,655)$, living outside of Lilongwe $(2.2 \%, 337 / 15,655)$, serious illness $(2.1 \%$, $321 / 15,121)$, drug/alcohol use $(0.1 \%, 14 / 15,086)$, and/or imprisonment $(0.2 \%, 26 / 15,083)$.

Patient acceptability of AHI testing was moderate; nearly two-thirds $(62.8 \%, 9280 / 14,755)$ of patients identified as eligible for testing consented to testing. Among consenting participants, 98.8\% (9171/9280) had results available; specimen clotting or having insufficient specimen volume for HIV RNA testing were the primary reasons for results not being confirmed.

Approximately half (52.2\%) of the 9280 of all persons tested for AHI were male (Table 1). Average age among all tested participants was 28.7 years [standard deviation (SD)

274 | www.jaids.com
8.3 years]. Overall, tested participants reported an average of 1.2 sexual partners in the last 3 months, and 12\% (1073/9280) of tested participants reported having $>1$ sexual partner in the last 3 months. Only 11\% (1030/9280) of participants reported condom use at last sex.

\section{Acutely Infected Persons vs Confirmed HIV-Negative}

We identified 59 persons with AHI, a prevalence of $0.64 \%$ (59/9171) among persons testing seronegative. The prevalence of AHI was similar by gender $[0.7 \%(34 / 4851)$ male vs $0.6 \%(25 / 4429)$ female, $P=0.4$; Table 2]. Seven additional persons had detectable HIV RNA but were considered false negative rapid antibody tests based upon repeat antibody testing of surplus specimen. Discordant rapid antibody tests were rare in the study population $(0.45 \%, 70 / 15,655)$, but persons with AHI were more likely to have discordant antibody test results compared with persons without AHI $(23.7 \%$ vs $0.4 \%, P<0.05$; Table 2$)$. We successfully traced 58/59 $(98.3 \%)$ persons with AHI within 21 days of testing using the locator/contact forms completed at the time of testing.

Median VL for persons with AHI was 758,050 copies per milliliter [interquartile range (IQR): 34,984-10,000,000]; the average VL was 3,304,732 copies per milliliter (SD $4,223,083)$. Median VL was lower among those with discordant rapid antibody tests $(276,980$ copies/mL, IQR: 13,780 $750,000)$ compared to persons with concordant negative results (1,838,060, IQR: $60,820-10,000,000, P=0.03)$. Mean VL was also lower among persons with discordant results $(423,292$ vs $4,201,179, P=0.001)$. Extremely high viral loads were common: $47 \%(28 / 59)$ had a VL $\geq 1,000,000$ copies per milliliter and $25 \%(15 / 59)$ had a VL $\geq 10,000,000$ copies per milliliter (upper limit of detection).

Persons with AHI were similar to those without AHI in terms of average age (28.2 vs 28.8 years, $P=0.3)$ and proportion male $(57.6 \%$ vs $52.2 \%, P=0.4)$. Persons with AHI had higher mean numbers of sexual partners in the last 3 months than persons without AHI (1.6 vs $1.1, P=0.1)$, and were more likely to have had $>1$ partner in the last 3 months (23.7\% vs $11.5 \%, P<0.01)$. Genital sores/ulcers were more common among persons with AHI $(42.4 \%$ vs $20.6 \%, P<$ 0.01). Persons with AHI were more likely to report having used a condom at last sex $(20.7 \%$ vs $11.9 \%, P=0.04)$. Symptoms frequently associated with AHI were more common among persons with AHI than those without AHI, including fever $(23.7 \%$ vs $8.7 \%, P<0.01)$, diarrhea $(10.2 \%$ vs $4.3 \%$, $P=0.03)$, and body aches $(28.8 \%$ vs $12.5 \%, P<0.01)$.

\section{STI vs HTC-AHI Prevalence, Risk Behaviors, and Clinical Symptoms}

STI clinic patients were more likely than HTC patients to be eligible for AHI testing [96.9\% (6456/6664) vs $92.3 \%$ (8299/8991), $P<0.01]$. In terms of AHI testing acceptability, screened and eligible participants at STI clinics were more likely to accept AHI testing compared with eligible participants at HTC clinics [65.9\% (4255/6456) vs 60.6\% (5025/ 8299), $P<0.01]$.

Copyright (C) 2015 Wolters Kluwer Health, Inc. All rights reserved. 


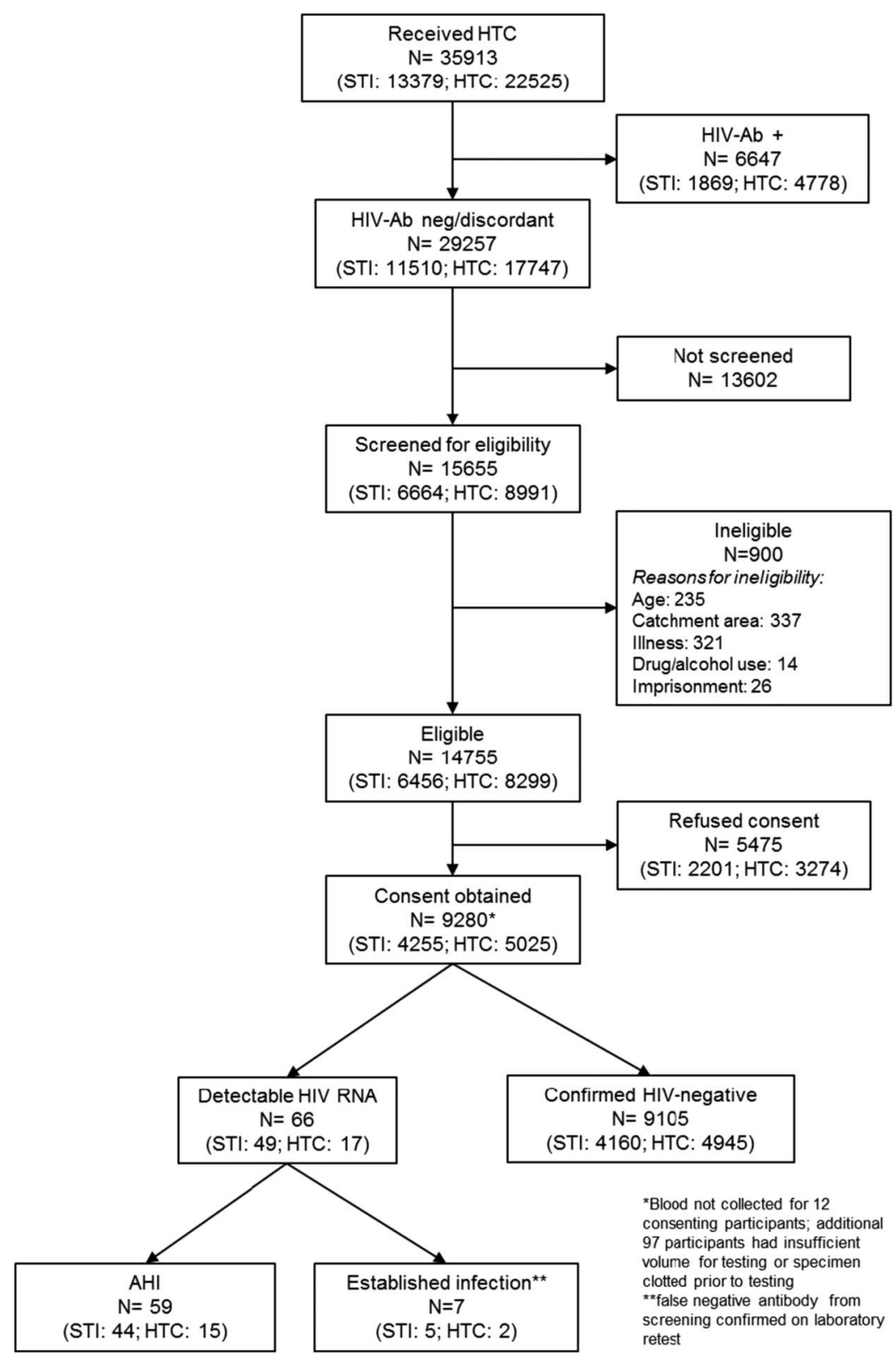

FIGURE 1. STI and HTC clinic patron eligibility and testing for AHI. All persons receiving HTC between June, 2012 and January, 2014 were recorded in HTC ledgers. Persons who were antibody negative or discordant (1 HIV-positive and 2 HIV-negative rapid antibody tests) were eligible to be screened for AHI testing. Approximately half were screened for study eligibility and, among those screened, approximately $94 \%$ were eligible for $\mathrm{AHI}$ testing. Among eligible persons, nearly two-thirds consented to AHI testing and HIV RNA results were available for nearly $99 \%$. Of persons with RNA results available, 66 had detectable RNA; 7 were false negative antibody based on repeat antibody screening and 59 had $\mathrm{AHI}$. Ab, antibody.

Among 59 persons with AHI, 44 (75\%) were tested at an STI clinic although only $46 \%$ of all persons tested were from an STI clinic. The AHI prevalence at STI clinics was $1.0 \%(44 / 4255)$, substantially higher than the AHI prevalence at HTC clinics $(0.3 \%, 15 / 5025, P<0.01$ Table 1$)$. Gender breakdown among persons with AHI was similar across sites [59\% (26/44) male (STI) vs 53\% (8/15) male (HTC)]. Persons with AHI accounted for 2.3\% (44/1913) of all HIV infections identified in STI clinics vs $0.31 \%(15 / 4793)$ of HIV-infected persons at HTC clinics, although these figures underestimate the true proportion of HIV attributable to AHI in the clinics because many HIV-seronegative patients were not screened for AHI.

VLs were higher among persons diagnosed in STI compared with HTC clinics (median VL 1,000,000 copies/mL,
STI vs 153,125 copies $/ \mathrm{mL}$, HTC, $P=0.2$; mean VL 3,522,480 copies/mL STI vs 2,666,005 copies/mL, HTC, $P=0.5$ ) (Fig. 2). Over half of persons with AHI from STI clinics had a VL $\geq 1,000,000$ copies per milliliter (52.3\%) compared with onethird of persons with AHI at HTC clinics $(P=0.2)$. Over onequarter $(27.3 \%)$ of persons with AHI from STI clinics had a VL $\geq 10,000,000$ copies per milliliter, compared to one-fifth of those with AHI at HTC clinics $(P=0.6)$. One-fifth $(20.5 \%)$ of persons with AHI from STI clinics had discordant rapid antibody test results at screening, compared with one-third of persons with AHI from HTC clinics $(P=0.3)$.

Patients tested at STI and HTC clinics had similar mean number of sexual partners in the last 3 months (1.1 vs 1.2 , $P=0.8$ ) (Table 3). Persons with AHI tested at STI clinics had fewer sexual partners, on average, compared with persons 
TABLE 1. Demographics, Risk Behavior, and Acute HIV at STI and HTC Clinics, All Screened $(\mathrm{N}=9280)$

\begin{tabular}{|c|c|c|}
\hline & $\begin{array}{c}\text { STI }(n=4255) \\
n(\%)\end{array}$ & $\begin{array}{c}\text { HTC }(n=5025), \\
n(\%)\end{array}$ \\
\hline \multicolumn{3}{|l|}{ Age* } \\
\hline $18-24$ & $1622(38.1)$ & $179(34.2)$ \\
\hline $25-34$ & $1906(44.8)$ & $2186(43.5)$ \\
\hline $35-44$ & $562(13.2)$ & $783(15.6)$ \\
\hline$\geq 45$ & $165(3.9)$ & $337(6.7)$ \\
\hline \multicolumn{3}{|l|}{ Sex* } \\
\hline Male & $2001(47.0)$ & $2850(56.7)$ \\
\hline Female & $2254(53.0)$ & $2175(43.3)$ \\
\hline \multicolumn{3}{|c|}{ No. partners (last $3 \mathrm{mo}$ ) } \\
\hline 0 & $217(5.1)$ & 599 (11.9) \\
\hline 1 & 3559 (83.6) & $3832(76.3)$ \\
\hline 2 & $329(7.7)$ & $424(8.4)$ \\
\hline 3 & $96(2.3)$ & $98(2.0)$ \\
\hline$\geq 4$ & $54(1.3)$ & $72(1.4)$ \\
\hline \multicolumn{3}{|c|}{ Partners (last $3 \mathrm{mo}$ ) } \\
\hline$\leq 1$ partner & 3776 (88.7) & $4431(88.2)$ \\
\hline$>1$ partner & $479(11.3)$ & $594(11.8)$ \\
\hline \multicolumn{3}{|l|}{ Condom use* } \\
\hline Yes & $362(8.8)$ & $668(14.8)$ \\
\hline No & 3738 (91.2) & $3839(88.2)$ \\
\hline \multicolumn{3}{|c|}{$\begin{array}{l}\text { Symptoms of genital ulcer } \\
\text { disease* }\end{array}$} \\
\hline Yes & $1710(40.2)$ & $209(4.2)$ \\
\hline No & $2545(59.8)$ & $4816(95.8)$ \\
\hline \multicolumn{3}{|c|}{ Discordant antibody results } \\
\hline Yes & $26(0.4)$ & $44(0.5)$ \\
\hline No & $6638(99.6)$ & 8947 (99.5) \\
\hline \multicolumn{3}{|c|}{ Acutely infected* } \\
\hline Yes & $44(1.0)$ & $15(0.30)$ \\
\hline No & $4211(99.0)$ & $5010(99.7)$ \\
\hline
\end{tabular}

with AHI tested at HTC clinics (1.4 partners STI vs 2.4 partners HTC, $P=0.05)$. Despite similar proportions reporting $>1$ partner in the last 3 months between clinic types in the entire screened study population $(11.3 \%$ STI vs $11.8 \%$ HTC, $P=0.4), 18.2 \%$ of persons with AHI at STI clinics reported $>1$ partner in the last 3 months, compared with $40 \%$ of persons with AHI at HTC clinics $(P=0.09)$. Men with AHI at STI clinics reported a mean of 1.7 partners, and over $30 \%$ reported having multiple partners in the last 3 months, whereas no women with AHI at STI clinics reported having more than 1 sexual partner in the last 3 months.

STI clinic patients were somewhat more likely to report symptoms than HTC clinic patients (fever $18.2 \%$ vs $40.0 \%$, $P=0.09$; diarrhea $6.8 \%$ vs $20.0 \%, P=0.2$; body aches $25.0 \%$ vs $40.0 \%, P=0.3)$. Not surprisingly, genital sores/ulcers were more common among persons tested at STI clinics compared with persons tested at HTC clinics $(40.2 \%$ vs $4.2 \%, P<$ 0.01). Among persons with AHI, those tested at STI clinics were more likely to report genital sores compared with those tested at HTC clinics $(54.6 \%$ vs $6.7 \%, P<0.01)$.

276 | www.jaids.com
TABLE 2. Demographics, Risk Behavior, and Testing Outcomes Comparing Persons With $\mathrm{AHI}$ to Persons Without AHI, All Screened $(\mathrm{N}=9280)$

\begin{tabular}{|c|c|c|}
\hline & $\begin{array}{c}\text { AHI (n= 59), } \\
\text { n (\%) }\end{array}$ & $\begin{array}{c}\text { No AHI }(n=9221) \text {, } \\
n(\%)\end{array}$ \\
\hline \multicolumn{3}{|l|}{ Age } \\
\hline $18-24$ & $24(40.7)$ & $3317(36.0)$ \\
\hline $25-34$ & $25(42.4)$ & $4067(44.1)$ \\
\hline $35-44$ & 7 (11.9) & $1338(14.5)$ \\
\hline$\geq 45$ & $3(5.1)$ & $499(5.4)$ \\
\hline \multicolumn{3}{|l|}{ Sex } \\
\hline Male & $34(57.6)$ & $4817(52.2)$ \\
\hline Female & $25(42.4)$ & $4404(47.8)$ \\
\hline \multicolumn{3}{|c|}{ No. partners (last $3 \mathrm{mo}$ ) } \\
\hline 0 & $1(1.7)$ & $815(8.8)$ \\
\hline 1 & $44(74.6)$ & 7347 (79.7) \\
\hline 2 & 7 (11.9) & $746(8.1)$ \\
\hline 3 & $1(1.7)$ & $193(2.1)$ \\
\hline$\geq 4$ & $6(10.2)$ & $120(1.3)$ \\
\hline \multicolumn{3}{|c|}{ Partners (last $3 \mathrm{mo}$ )* } \\
\hline$\leq 1$ partner & $45(76.3)$ & $8162(88.5)$ \\
\hline$>1$ partner & $14(23.7)$ & $1059(11.5)$ \\
\hline \multicolumn{3}{|l|}{ Condom use* } \\
\hline Yes & $12(20.7)$ & $1018(11.9)$ \\
\hline No & $46(79.3)$ & $7531(88.1)$ \\
\hline \multicolumn{3}{|c|}{$\begin{array}{l}\text { Symptoms of genital ulcer } \\
\text { disease* }\end{array}$} \\
\hline Yes & $25(42.4)$ & $1894(20.5)$ \\
\hline No & $34(57.6)$ & $7327(79.5)$ \\
\hline \multicolumn{3}{|c|}{ Discordant antibody results* } \\
\hline Yes & $14(23.7)$ & $41(0.4)$ \\
\hline No & $45(76.3)$ & $9180(99.6)$ \\
\hline
\end{tabular}

\section{AHI Testing Risk Score Performance}

Overall, the risk score algorithm had sensitivity of $71 \%$ and specificity of $73 \%$ for identifying persons with AHI. Use of this risk score in our population would have resulted in testing only $27.6 \%(2559 / 9280)$ of all persons, and would have identified $71.2 \%(42 / 59)$ of persons with AHI.

In the STI clinics, algorithm sensitivity was unchanged (71\%), specificity was $54 \%$, and $46.4 \%(1972 / 4255)$ of participants met the testing threshold. Thus in STI clinics, by conducting AHI testing on approximately half of all patients $(46 \%)$, we would have identified $70.4 \%$ (31/44) of all persons with AHI. In the HTC clinics, sensitivity was $73 \%$ and specificity improved substantially to $89 \%$ with only $11.7 \%$ of HTC participants meeting the threshold score. However, testing $\sim 12 \%$ of all HTC patients would have identified $73 \%(11 / 15)$ of all persons with AHI.

\section{DISCUSSION}

AHI testing was largely acceptable when implemented at STI and HTC clinics in Lilongwe. In these high-volume settings, more than half of seronegative patients were screened for study eligibility and nearly two-thirds of eligible

Copyright (C) 2015 Wolters Kluwer Health, Inc. All rights reserved. 


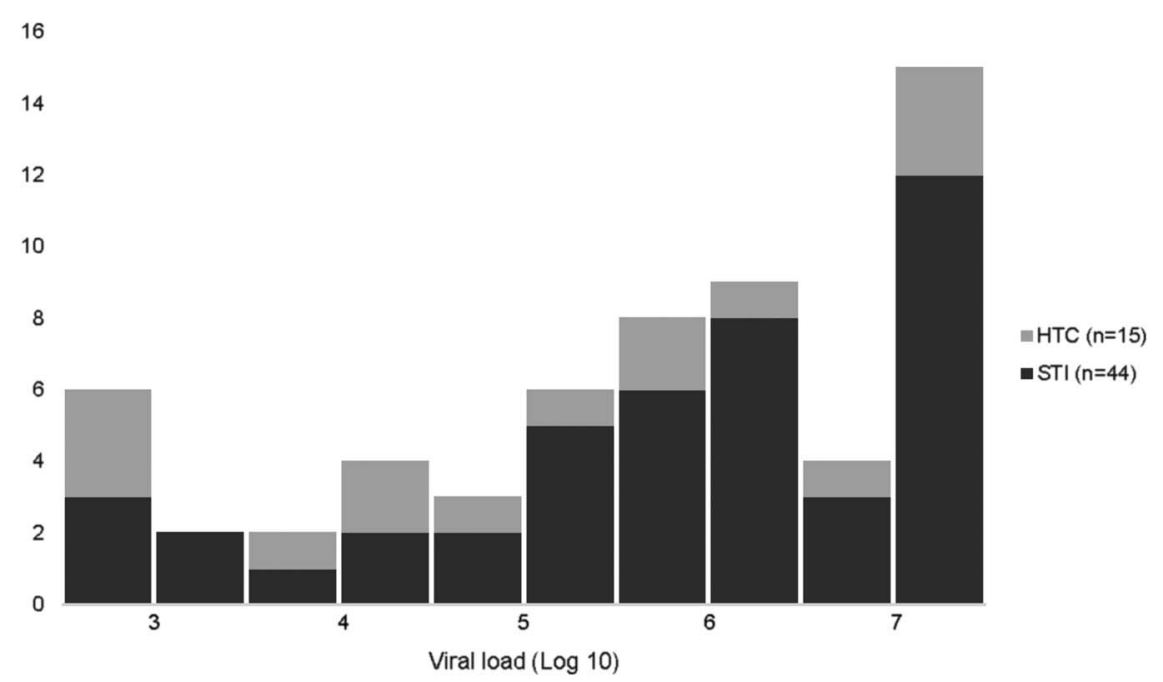

FIGURE 2. Viral loads among persons with AHI. Median and mean VL for all persons with $\mathrm{AHI}$ was 758,050 copies per milliliter (IQR: 34,984-10,000,000) and 3,304,732 copies per milliliter (SD 4,223,083), respectively. VLs tended to be higher among persons diagnosed in STI clinics, compared with HTC clinics (median VL $1,000,000$ copies/mL vs 153,125 copies/ $\mathrm{mL}, P=0.2$; mean VL $3,522,480$ copies $/ \mathrm{mL}$ vs $2,666,005$ copies $/ \mathrm{mL}, P=0.5$ ).

Viral load $(\log 10)$

patients consented to AHI testing. Almost all persons with AHI were successfully traced and informed of their HIV status. The potential for transmission in this group of acutely infected persons was remarkable; patients had extremely high VLs, a substantial proportion presented with concomitant genital ulcer disease, and the majority reported recent unprotected sexual encounters.

This study represents one of the largest prospective screening initiatives for AHI among persons presenting to STI and HTC clinics in sub-Saharan Africa. Although AHI prevalence has been evaluated in STI clinics, ${ }^{23,24,26,32}$ to our knowledge, routine screening has not been incorporated into an HTC setting previously. We observed important differences between these 2 populations in terms of AHI prevalence and potential infectiousness-both of which were higher among STI clinic patients. The clinic differences may provide some insight into transmission dynamics. The increased proportion of discordant test results and the slightly lower VL among persons with AHI at HTC suggests that these persons are detected at a slightly longer interval after

TABLE 3. Demographics, Risk Behaviors, and Testing Outcomes by Clinic Type and Sex, All AHI $(N=59)$

\begin{tabular}{|c|c|c|c|c|c|c|}
\hline & \multicolumn{3}{|c|}{ STI } & \multicolumn{3}{|c|}{ HTC } \\
\hline & $\begin{array}{c}\text { Overall }(n=44), \\
n(\%)\end{array}$ & $\begin{array}{c}\text { Male }(n=26), \\
n(\%)\end{array}$ & $\begin{array}{c}\text { Female }(n=18), \\
n(\%)\end{array}$ & $\begin{array}{c}\text { Overall }(n=15), \\
n(\%)\end{array}$ & $\begin{array}{c}\text { Male }(n=8), \\
\text { n }(\%)\end{array}$ & $\begin{array}{c}\text { Female }(\mathbf{n}=7), \\
n(\%)\end{array}$ \\
\hline \multicolumn{7}{|l|}{ Mean age, yrs } \\
\hline $18-24$ & $18(40.9)$ & $8(30.8)$ & $10(55.6)$ & $6(40.0)$ & $3(37.5)$ & $3(42.1)$ \\
\hline $25-34$ & $19(43.2)$ & $14(53.9)$ & $5(27.8)$ & $6(40.0)$ & $4(50.0)$ & $2(28.6)$ \\
\hline $35-44$ & $5(11.4)$ & $2(7.7)$ & $3(15.7)$ & $2(13.3)$ & $1(12.5)$ & $1(14.3)$ \\
\hline$\geq 45$ & $2(4.6)$ & $2(7.7)$ & 0 & $1(6.7)$ & 0 & $1(14.3)$ \\
\hline \multicolumn{7}{|c|}{ No. partners (last $3 \mathrm{mo}$ ) } \\
\hline 0 & 0 & 0 & 0 & $1(6.7)$ & 0 & $1(14.3)$ \\
\hline 1 & $36(81.8)$ & $18(69.2)$ & $18(100)$ & $8(53.3)$ & $4(50.0)$ & $5(57.1)$ \\
\hline 2 & $5(11.4)$ & $5(19.2)$ & 0 & $2(13.3)$ & $2(25.0)$ & 0 \\
\hline 3 & $3(6.8)$ & $3(11.5)$ & 0 & $1(6.7)$ & $1(12.5)$ & 0 \\
\hline$\geq 4$ & 0 & 0 & 0 & $3(20.0)$ & $1(12.5)$ & $2(28.6)$ \\
\hline \multicolumn{7}{|c|}{ Partners (last $3 \mathrm{mo}$ )* } \\
\hline$\leq 1$ partner & $36(81.8)$ & $18(69.2)$ & $18(100)$ & $9(60.0)$ & $4(50.0)$ & $5(71.4)$ \\
\hline$>1$ partner & $8(18.2)$ & $8(30.8)$ & 0 & $6(40.0)$ & $4(50.0)$ & $2(28.6)$ \\
\hline \multicolumn{7}{|l|}{ Condom use } \\
\hline Yes & 7 (15.9) & $4(15.4)$ & $3(16.7)$ & $5(35.7)$ & $3(37.5)$ & $2(33.3)$ \\
\hline No & $37(84.1)$ & $22(84.6)$ & $15(83.3)$ & $9(64.3)$ & $5(62.5)$ & $4(66.7)$ \\
\hline \multicolumn{7}{|c|}{$\begin{array}{l}\text { Discordant antibody } \\
\text { results }\end{array}$} \\
\hline Yes & $9(20.4)$ & $4(15.4)$ & $5(27.8)$ & $5(33.3)$ & $1(12.5)$ & $4(57.1)$ \\
\hline No & 35 (79.6) & $22(84.6)$ & $13(72.2)$ & $10(66.7)$ & $7(87.5)$ & $3(42.9)$ \\
\hline
\end{tabular}

$* P<0.05$ for STI clinic, male vs female. 
acquisition, once the peak viremia has subsided and after more sensitive antibody tests can detect antibodies, but before they are concordant positive for antibodies. Conversely, persons with AHI from STI clinics appear to be presenting earlier, possibly in response to STI-related symptoms associated with an infection acquired around the time of HIV infection. Based on higher VLs and the presence of genital sores/ulcers, persons with AHI tested at STI clinics may be more likely than their acutely-infected counterparts tested at HTC clinics to transmit HIV.

Risk behaviors at both clinic types, including infrequent condom use and multiple sexual partners, reinforce the importance of screening for AHI to reduce forward transmission. Although behaviors among the entire screened population at STI and HTC clinics were similar, differences by AHI were observed. In the STI clinics, all women with AHI reported only 1 partner within the last 3 months. One possible explanation for this is that women were infected by their primary partner as a consequence of a concomitant STI in the woman or her partner. Alternatively, women may be more likely to underreport multiple partners. The number of HIV-negative partners is critical to appreciating the role of AHI in transmission events, and detailed behavioral data from the pilot randomized controlled trial, forming the basis of the current study, will help elucidate true partnership patterns among these women.

Targeting AHI testing based on risk behaviors and select acute retroviral symptoms may improve AHI screening efficiency and could be a more cost-effective option for AHI screening in high-prevalence, resource-limited settings. After symptom-guided testing in Uganda, Mozambique, Kenya, and South Africa, $1 \%-3 \%$ of adults in outpatient settings had AHI. ${ }^{19-22}$ We evaluated performance of a previously validated risk score that weighs symptoms, antibody results, and risk behaviors to prioritize screening for persons at greatest risk of AHI. ${ }^{26,33}$ Despite having been developed in the context of an STI clinic, the algorithm performed better when applied to HTC patients; we could identify $>70 \%$ of all new infections by screening only $12 \%$ of all HTC clients. This observed efficiency may be driven by the higher frequency of discordant rapid tests among HTC patients, a heavilyweighted component of the risk score. Discordant antibody results had a $24 \%$ positive-predictive value for $\mathrm{AHI}$, similar to that observed in previous studies of AHI in this population. ${ }^{26}$ One advantage of the risk score is that it goes beyond symptoms, incorporating risk behaviors and biologic markers (antibody test discordancy) that are often associated with AHI. These elements are critical in light of the nontrivial proportion of patients presenting with atypical symptoms of AHI. ${ }^{34}$ However, the value of the risk score should be evaluated in the context in which it was tested, namely high prevalence settings in the background of endemic HIV. The utility of targeted screening using symptoms of early infection may not be realized in low-prevalence settings. ${ }^{35}$ Although we did not capture costs associated with AHI screening in this study, use of targeted screening may be suitable in HTC clinics, containing costs by improving efficiency of $\mathrm{AHI}$ screening in this population with a lower prevalence of AHI.

As the first report of AHI screening from HTC, there is no comparator by which to evaluate change in AHI preva-

278 | www.jaids.com lence in this population. In STI clinics, despite a decline in AHI prevalence, the proportion of all HIV diagnoses attributable to AHI appears to be steady or higher compared with previous studies. Historically, AHI rates among STI clinic patients in Malawi ranged from $2 \%$ to $5 \%$ of HIV-seronegative patients, accounting for 3\%-5\% of new HIV diagnoses. ${ }^{2,24}$ In this study, among screened HIV-seronegative STI patients, $1.0 \%$ were acutely infected, accounting for $2.3 \%$ of new HIV diagnoses. However, we were only able to screen one-third of the seronegative patients. At the STI clinic, if we extrapolate the observed rates of eligibility and AHI to the approximately 13,600 seronegative persons who were not screened for the study, and the approximately 5500 who did not consent, we anticipate 70 persons with AHI were missed among all seronegative patients. Under these assumptions, acute infection may account for up to $5.7 \%$ of new HIV diagnoses at STI clinics.

AHI screening acceptability observed in this study is likely a gross underestimate of true testing acceptability in light of the testing occurring in the research setting. Although two-thirds of all eligible patients accepted testing, we do not have information regarding why eligible patients refused $\mathrm{AHI}$ testing. Eligible patients at STI clinics were more likely to accept AHI testing compared with their HTC counterparts. This difference could reflect differential perceived risk of AHI between these 2 patient populations. Alternatively, the difference may be due to superior prestudy sensitization by counselors at STI clinics, many of whom had previous experience screening for AHI. In this study, accepting AHI testing meant committing to the additional time necessary for provision of consent and data collection. Consent and data collection may have also been a barrier for eligibility assessment; we observed a lower-than-desired rate of screening for study eligibility with just over half of all HIVseronegative patients evaluated. However, in that we did not use symptom-based assessments before eligibility assessment; the seronegative patients who were assessed for study eligibility were not any more or less likely to have AHI. Although critical elements in the context of our investigation, consent and data collection barriers are unlikely to be obstacles to AHI testing outside of a research setting. We would expect higher rates of testing acceptability if such screening was incorporated into routine algorithms.

Rapid specimen testing and patient notification of $\mathrm{AHI}$ status is critical. Using pooled HIV RNA PCR, nearly $99 \%$ of specimens were tested within 7 days of screening. The 21-day protocol-permitted window may miss peak viremia, but is still within the 10-12 week period of increased infectiousness and represents a significant improvement over the current standard of care. Nonetheless, faster testing turnaround time may reduce transmissions occurring between screening and result availability. Unfortunately, no effective point-of-care AHI tests are available. ${ }^{36-38}$ Fourth generation enzyme linked immunoassays may be a suitable and cost-effective alternative to HIV RNA, ${ }^{39}$ but still require expensive and extensive laboratory infrastructure. The serum volume required by these assays precludes fingerstick collection, in contrast to RNA testing which can pool small volumes of serum. ${ }^{40,41}$ Current HIV testing algorithms in Malawi use exclusively fingerstick; 
counselors are rarely trained in venous phlebotomy. Despite the complexity of HIV RNA PCR, standard centralized labs serving numerous clinics within a city or region may facilitate use of this technology, yielding greater daily screening volume, and enabling larger pool sizes for more efficient testing. ${ }^{41}$ HIV RNA will play a prominent role in AHI screening until fourth generation assays can test with smaller whole blood volume, or point-of-care devices demonstrate their suitability for use in the field.

Even without point-of-care options, notification of AHI results was efficient. Among the 46 patients who were enrolled into the subsequent pilot study, the median time from screening to enrollment was 7 days (data not shown), indicating that most patients were tested and informed of their AHI results within 7 days. The tracing efforts in this study included a team of community educators who used patientprovided information to locate acutely-infected persons. Resource-utilization for these efforts was not captured. Although generalizability to more rural areas merits further evaluation, use of community educators may be scalable.

We have demonstrated that AHI testing can be incorporated into routine HIV testing procedures and may be an acceptable add-on to existing antibody-based testing algorithms. AHI prevalence was significantly higher among STI clinic patients compared with HTC clinic patients, and accounts for a substantial proportion of all new HIV diagnoses. We demonstrated that targeted screening for HTC patients using a risk score is an efficient means of identifying acute infection, and would help focus screening resources in this lower prevalence population. Based on our results, AHI screening in both STI and HTC clinic settings may be warranted in high-incidence settings, such as Malawi, and should be made a priority for HIV prevention. Additional considerations, including cost-effectiveness and feasibility outside of a controlled trial setting, will be critical to design and implement sound AHI-focused testing policies. We recommend targeted AHI screening for HTC patients and universal AHI screening for STI patients.

\section{ACKNOWLEDGMENTS}

The authors acknowledge the numerous HIV testing and counseling staff at Lighthouse and UNC Project clinics who assisted in study activities.

\section{REFERENCES}

1. Pilcher CD, Shugars DC, Fiscus SA, et al. HIV in body fluids during primary HIV infection: implications for pathogenesis, treatment and public health. AIDS. 2001;15:837-845.

2. Vaidya NK, Ribeiro RM, Miller CJ, et al. Viral dynamics during primary simian immunodeficiency virus infection: effect of time-dependent virus infectivity. J Virol. 2010;84:4302-4310.

3. Ma ZM, Stone M, Piatak M Jr, et al. High specific infectivity of plasma virus from the pre-ramp-up and ramp-up stages of acute simian immunodeficiency virus infection. J Virol. 2009;83:3288-3297.

4. Pilcher CD, Joaki G, Hoffman IF, et al. Amplified transmission of HIV1: comparison of HIV-1 concentrations in semen and blood during acute and chronic infection. AIDS. 2007;21:1723-1730.

5. Morrison CS, Demers K, Kwok C, et al. Plasma and cervical viral loads among Ugandan and Zimbabwean women during acute and early HIV-1 infection. AIDS. 2010;24:573-582.
6. Brenner BG, Roger M, Routy JP, et al. High rates of forward transmission events after acute/early HIV-1 infection. $J$ Infect Dis. 2007;195:951-959.

7. Cohen MS, Shaw GM, McMichael AJ, et al. Acute HIV-1 infection. N Engl J Med. 2011;364:1943-1954.

8. Miller WC, Rosenberg NE, Rutstein SE, et al. Role of acute and early HIV infection in the sexual transmission of HIV. Curr Opin HIV AIDS. 2010;5:277-282.

9. Pilcher CD, Tien HC, Eron JJ Jr, et al. Brief but efficient: acute HIV infection and the sexual transmission of HIV. J Infect Dis. 2004;189: $1785-1792$.

10. Cohen MS, McCauley M, Gamble TR. HIV treatment as prevention and HPTN 052. Curr Opin HIV AIDS. 2012;7:99-105.

11. Powers KA, Ghani AC, Miller WC, et al. The role of acute and early HIV infection in the spread of HIV and implications for transmission prevention strategies in Lilongwe, Malawi: a modelling study. Lancet. 2011;378:256-268.

12. White PJ, Fox J, Weber J, et al. How many HIV infections may be averted by targeting primary infection in men who have sex with men? Quantification of changes in transmission-risk behavior, using an individual-based model. J Infect Dis. 2014;210(suppl 2):S594-S599.

13. Marks G, Crepaz N, Senterfitt JW, et al. Meta-analysis of high-risk sexual behavior in persons aware and unaware they are infected with HIV in the United States: implications for HIV prevention programs. $J$ Acquir Immune Defic Syndr. 2005;39:446-453.

14. Steward WT, Remien RH, Higgins JA, et al. Behavior change following diagnosis with acute/early HIV infection-a move to serosorting with other HIV-infected individuals. The NIMH Multisite Acute HIV Infection Study. III. AIDS Behav. 2009;13:1054-1060.

15. Gous N, Scott L, Perovic O, et al. Should South Africa be performing nucleic acid testing on HIV enzyme-linked immunosorbent assaynegative samples? J Clin Microbiol. 2010;48:3407-3409.

16. Juusola JL, Brandeau ML, Long EF, et al. The cost-effectiveness of symptom-based testing and routine screening for acute HIV infection in men who have sex with men in the USA. AIDS. 2011;25:1779-1787.

17. Karris MY, Anderson CM, Morris SR, et al. Cost savings associated with testing of antibodies, antigens, and nucleic acids for diagnosis of acute HIV infection. J Clin Microbiol. 2012;50:1874-1878.

18. Cragin L, Pan F, Peng S, et al. Cost-effectiveness of a fourth-generation combination immunoassay for human immunodeficiency virus (HIV) antibody and p24 antigen for the detection of HIV infections in the United States. HIV Clin Trials. 2012;13:11-22.

19. Bebell LM, Pilcher CD, Dorsey G, et al. Acute HIV-1 infection is highly prevalent in Ugandan adults with suspected malaria. AIDS. 2010;24: $1945-1952$.

20. Sanders EJ, Mugo P, Prins HA, et al. Acute HIV-1 infection is as common as malaria in young febrile adults seeking care in coastal Kenya. AIDS. 2014;28:1357-1363.

21. Serna-Bolea C, Munoz J, Almeida JM, et al. High prevalence of symptomatic acute HIV infection in an outpatient ward in southern Mozambique: identification and follow-up. AIDS. 2010;24:603-608.

22. Bassett IV, Chetty S, Giddy J, et al. Screening for acute HIV infection in South Africa: finding acute and chronic disease. HIV Med. 2011;12:46-53.

23. Pilcher CD, Price MA, Hoffman IF, et al. Frequent detection of acute primary HIV infection in men in Malawi. AIDS. 2004;18:517-524.

24. Fiscus SA, Pilcher CD, Miller WC, et al. Rapid, real-time detection of acute HIV infection in patients in Africa. $J$ Infect Dis. 2007;195:416-424.

25. Rutstein SE, Pettifor A, Phiri S, et al. Identifying persons with acute HIV infection in urban Malawi HIV testing and sexually transmitted infection clinics: an opportunity for HIV transmission prevention. Paper presented at: 20th International AIDS Conference; July 2014; Melbourne, Australia.

26. Powers KA, Miller WC, Pilcher CD, et al. Improved detection of acute HIV-1 infection in sub-Saharan Africa: development of a risk score algorithm. AIDS. 2007;21:2237-2242.

27. Global AIDS Response Progress Report: Malawi Progress report for 2013. Lilongwe: Ministry of Health; 2014.

28. Westreich DJ, Hudgens MG, Fiscus SA, et al. Optimizing screening for acute human immunodeficiency virus infection with pooled nucleic acid amplification tests. J Clin Microbiol. 2008;46:1785-1792.

29. Pilcher $\mathrm{CD}$, McPherson JT, Leone PA, et al. Real-time, universal screening for acute HIV infection in a routine HIV counseling and testing population. JAMA. 2002;288:216-221. 
30. Priddy FH, Pilcher CD, Moore RH, et al. Detection of acute HIV infections in an urban HIV counseling and testing population in the United States. J Acquir Immune Defic Syndr. 2007;44:196-202.

31. Pilcher CD, Fiscus SA, Nguyen TQ, et al. Detection of acute infections during HIV testing in North Carolina. $N$ Engl $J$ Med. 2005;352:1873-1883.

32. Wolpaw BJ, Mathews C, Chopra M, et al. Diagnosis and counselling of patients with acute HIV infection in South Africa. Sex Transm Infect. 2011;87:71-72.

33. Wahome E, Fegan G, Okuku HS, et al. Evaluation of an empiric risk screening score to identify acute and early HIV-1 infection among MSM in Coastal Kenya. AIDS. 2013;27:2163-2166.

34. Braun DL, Kouyos RD, Balmer B, et al. Frequency and spectrum of unexpected clinical manifestations of primary HIV-1 infection. Clin Infect Dis. 2015;61:1013-1021.

35. Wood E, Kerr T, Rowell G, et al. Does this adult patient have early HIV infection?: the Rational Clinical Examination systematic review. JAMA. 2014;312:278-285.

36. Conway DP, Holt M, McNulty A, et al. Multi-centre evaluation of the Determine HIV Combo assay when used for point of care testing in a high risk clinic-based population. PLoS One. 2014;9: e94062.

37. Duong YT, Mavengere $\mathrm{Y}$, Patel $\mathrm{H}$, et al. Poor performance of the Determine HIV-1/2 Ag/Ab combo fourth-generation rapid test for detection of acute infections in a National Household Survey in Swaziland. J Clin Microbiol. 2014;52:3743-3748.

38. Rosenberg NE, Kamanga G, Phiri S, et al. Detection of acute HIV infection: a field evaluation of the determine( $\mathrm{R}$ ) HIV-1/2 Ag/Ab combo test. J Infect Dis. 2012;205:528-534.

39. Long EF. HIV screening via fourth-generation immunoassay or nucleic acid amplification test in the United States: a cost-effectiveness analysis. PLoS One. 2011;6:e27625.

40. Hutchinson AB, Patel P, Sansom SL, et al. Cost-effectiveness of pooled nucleic acid amplification testing for acute HIV infection after thirdgeneration HIV antibody screening and rapid testing in the United States: a comparison of three public health settings. Plos Med. 2010;7:e1000342.

41. Krajden M, Cook D, Mak A, et al. Pooled nucleic acid testing increases the diagnostic yield of acute HIV infections in a high-risk population compared to 3rd and 4th generation HIV enzyme immunoassays. $J$ Clin Virol. 2014;61:132-137. 